\title{
Exact solution of a generalised model for surface deposition
}

\author{
J. A. N. Filipe*1 and G. J. Rodgers ${ }^{2}$ \\ 1 Department of Plant Sciences, University of Cambridge, Cambridge CB2 3EA, \\ UK and Biomathematics \& Statistics Scotland, The University of Edinburgh, \\ The King's Buildings (JCMB), Edinburgh EH9 3JZ, UK \\ 2 Department of Mathematical Sciences, Brunel University, Uxbridge, Middlesex UB8 3PH, UK
}

(Dated: 06 March, 2003)

\begin{abstract}
We consider a model for surface deposition in one dimension in the presence of both precursor-layer diffusion and desorption. The model is a generalisation that includes RSA, ARSA and growth-andcoalescence models as special cases. Exact solutions are obtained for the model in both its lattice and continuum version. Expressions are obtained for physically important quantities such as the surface coverage, average island size, mass-adsorption efficiency and the process efficiency. The connection between a limiting case of the model and epidemic models is discussed.
\end{abstract}

PACS numbers: 05.20.-y, 02.50.Cw, 82.20.Mj

Keywords: chemisorption, physisorption, desorption, diffusion, nucleation, coalescence

Random sequential adsorption (RSA) [1-3] is a model process by which particles are irreversibly deposited without overlap and without positional correlation onto a surface from a gas [4] or solution [5]. Once deposited the particles cannot move on the surface, nor desorb back into the fluid phase. The model describes a physical situation in which impenetrable particles interact weakly with one another and where diffusion and desorption are negligible. Many extensions of the RSA model have been proposed in order to include, for example, diffusion on or desorption from the substract $[3,6]$ and local rolling and rearrangement of particles [7].

Motivated by theoretical [8] and experimental [9] work, in [11] we introduced a model in which it is possible for the particles to diffuse on top of previously deposited particles. More precisely, in this model the deposition of a particle is attempted at a randomly selected position on the surface; if the position is full or partly occupied, then the incident particle diffuses along the top of the deposited particles until it finds a space large enough to accommodate it; then the particle is deposited instantly and irreversibly. In the language of surface chemistry $[4,10]$, this model describes systems in which particles can become physisorbed and diffuse in a precursor state, until they become chemisorbed at some later time. In [12] this process was called accelerated random sequential adsorption (ARSA). Exact results were obtained for the gap distribution function and for physically relevant quantities, such as the surface coverage, the average island size and the probabilities of island nucleation, growth and aggregation, in one dimension. The continuum version of the model was also studied. Later, these results were extended [13] to study the accelerated random sequential adsorption of particles on to a surface with random impurities.

More recently, a new application has been proposed for

\footnotetext{
*Corresponding author. E-mail address: jf263@cam.ac.uk
}

the ARSA model. In [14] the model was used to study a computer science algorithm called linear probing with hashing [15]. This is an efficient and widely used algorithm for inserting items into a table. The cost function of the algorithm is related to the number sites that a precursor-diffusing particle visits before being deposited.

In this paper we extend our previous results to include the possibility of desorption from the precursor state. In order to do so, we consider a generalised model for deposition which comprises RSA, ARSA and other processes as particular cases. This extension is motivated by the fact that the energy bond in the physisorbed state is weaker than that in the chemisorbed state, so particles can more easily desorb from the precursor state $[4,8,10]$. This situation corresponds, in the computer table-filling algorithm, to a probability of failure when the duration of the search operation is too long.

We consider the ARSA of $k$-mers (i.e., particles of size $k$ ) onto a linear lattice [11]. The rate of successful depositions following precursor diffusion is $D$ and, as usual, the rate of successful direct depositions on empty sites is set to 1 . The cases $D=0$ and $D=1$ correspond to standard RSA and ARSA $[11,12]$ respectively. The introduction of this parameter was first discussed in [11] although only the case $D=1$ was studied there. Allowing $D$ to vary between 1 and 0 accounts for additional possibilities: that an incoming molecule might be more likely to be scattered from an occupied region than from an empty region, or that once in the precursor state it might desorb before reaching an empty site where it can be deposited. The complementary case $D>1$ also has physical meaning. For example, it represents situations where molecules are more likely to stick to the substrate at the edge of growing clusters of identical molecules. The limit $D \rightarrow \infty$ corresponds to a special case of ARSA, with growth and coalescence (from an initial distribution of 'seeds' or impurities) but without subsquent nucleation. The interval $0 \leq D \leq 1$ is perhaps the most relevant to surface adsorption systems.

Let $C_{r}(t)$ denote the average density of gaps of length 
$r$ between occupied regions at time $t$ (distribution of gap sizes). By average density, we mean the total number of occurrences of a gap of size $r$, divided by the system size and averaged over the distribution of stochastic realisations from an empty substrate up to time $t$. As usual, $t$ measures (in arbitrary time units) the number of deposition attempts divided by the system size.

The evolution equations which the set of $C_{r}(t)$ obeys were derived in [12] for the case $D=1$. Extension to general rate $D$ is obtained by multiplying by $D$ the terms representing precursor-mediated deposition; this gives

$$
\begin{aligned}
\frac{d C_{r}(t)}{d t}= & 2 \sum_{s=0}^{\infty} C_{r+s+k}(t)-[r-(k-1)] C_{r}(t) \\
& +D q(t)\left[C_{r+k}(t)-C_{r}(t)\right]
\end{aligned}
$$

for $r \geq k$, where the four terms represent the creation and destruction of a gap of size $r$ by direct and by precursormediated deposition. For $r<k$ there are no destruction (negative) terms, and $C_{r}(t)$ is easily expressed as an integral involving the solution of Eqn. (1). The quantity

$$
q(t)=\left[1-\sum_{r=k}^{\infty}[r-(k-1)] C_{r}(t)\right] /\left[\sum_{r=k}^{\infty} C_{r}(t)\right]
$$

is the average number of positions (per gap with $r \geq k$ ) where a particle can physisorb before diffusing towards an edge site where chemisorption takes place. Corresponding equations for the average density of occupied regions of length $r$ (islands) were derived in [11]. These equations do not have closed form, as a consequence of the process of island coalescence, so they were solved in [11] using truncation approximations.

Despite their nonlinearity, Eqs. (1) can be solved for general $k$ using a similar method as in $[12,13,16,18]$. As argued and verified by simulation in $[12,13]$, we expect the size of the gaps with $r \geq k$ to be independent of the evolving island structure. This is because new gaps form (i.e., islands nucleate) by direct, random deposition. This leads to the assumption that gaps of size $r \geq k$ have the Poisson distribution, $C_{r}(t)=A(t) \exp [-(r-k) t]$, where $A(t)$ is to be determined. For initial conditions $C_{r}(0)=0$ and $\lim _{t \rightarrow 0} \sum_{r=1}^{\infty} r C_{r}(t)=1$, corresponding to an empty substrate, the solution is

$$
C_{r}(t)=\frac{\left[1-e^{-t}\right]^{2}[G(t)-D]}{2-D} e^{-(r-k) t}
$$

for $r \geq k$, and

$$
C_{r}(t)=\int_{0}^{t} d u\left[1-e^{-u}\right] G(u) e^{-r u}
$$

for $r<k$, with

$$
G(t)=\frac{2-D \int_{0}^{t} d u F(u)}{F(t)}
$$

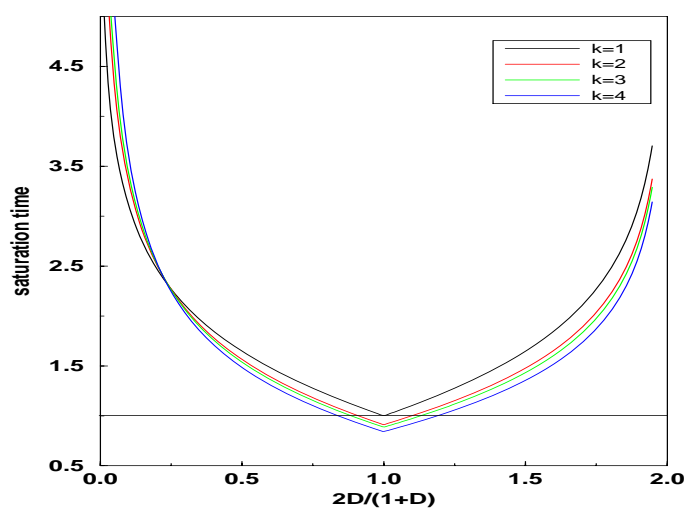

FIG. 1: Rescaled saturation time $k t_{c}$.

and

$$
F(t)=\exp \left[(1-D) t+(2-D) \sum_{r=1}^{k-1} \frac{1-e^{-r t}}{r}\right] .
$$

This solution holds for $t \leq t_{c}$, where $t_{c}$ is the saturation time, after which no more particles can be deposited; $t_{c}$ is a measure of the number of deposition attempts made until saturation (in multiples of $k$ lattice sites). The saturation time $t_{c}$ is determined by $C_{r}\left(t_{c}\right)=0$ for all $r \geq k$, and obeys the equation $G\left(t_{c}\right)=D$, or

$$
F\left(t_{c}\right)+\int_{0}^{t_{c}} d u F(u)=2 / D .
$$

Fig. 1 plots $k t_{c}$, the total mass of particles that have collided with the substrate until saturation, against $D$ for various particle sizes $k$, from $D=0$ (RSA), through $D=1$ (ARSA), to $D=\infty$ (on the right). There is a point $\left(D^{*} \approx 0.125\right)$ at which all curves approximately meet; for $D>D^{*}$ there is a small decrease of $k t_{c}$ with $k$ (which is maximum around $D=0.5$ ); for $D<D^{*}$ there is a large increase with $k$. This behaviour originates from different trade-offs between the direct and precursormediated deposition mechanisms, each of which yields distinct forms of deposition failure. In the RSA limit $(D \rightarrow 0)$ the saturation time has a logarithmic divergence $t_{c} \simeq \ln (1 / D)$, as revealed by inspection of $(7)$.

The central quantity in this system is the fraction of surface occupied, or surface coverage, $\theta(t)$. The coverage is directly related to the distribution of island sizes, which is not known exactly. Fortunately, thanks to the binary nature of the problem (sites are either empty or occupied), $\theta(t)$ also relates to the distribution of gap sizes, $\theta(t)=1-\sum_{r=1}^{\infty} r C_{r}(t)$. Direct substitution of Eqs.(3)(4) into this definition is cumbersome; it is much simpler to use the relation

$$
\frac{d \theta}{d t}=k\left\{P_{k}(t)+D\left[1-P_{k}(t)\right]\right\}
$$

between the sticking probability and $P_{k}(t)=\sum_{r=k}^{\infty}[r-$ $(k-1)] C_{r}(t)$, the probability that a $k$-mer lands directly 


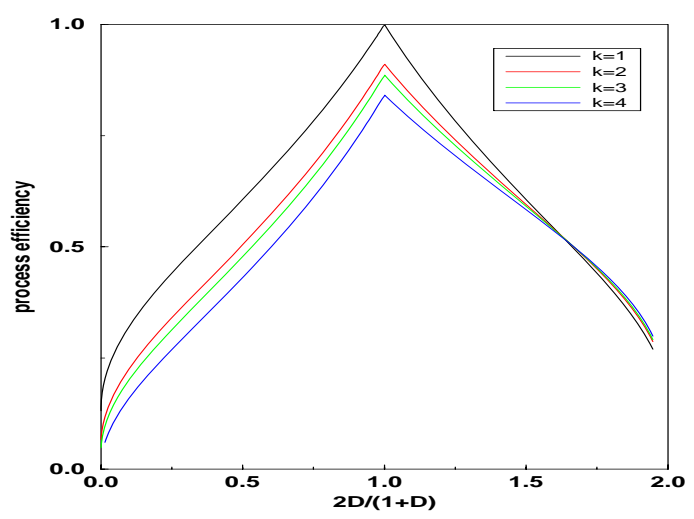

FIG. 2: Process efficiency $\epsilon$.

on an empty interval of size $k$. This gives

$$
\theta(t)=\frac{k}{2-D}\left[D t+(1-D) \int_{0}^{t} d u G(u)\right]
$$

which behaves asymptotically as

$$
\theta(t)=\theta\left(t_{c}\right)-k D\left(t_{c}-t\right)+O\left(t_{c}-t\right)^{2}
$$

in the approach to saturation. Also of interest is the average island size $\langle L(t)\rangle$ (and the average number of particles per island $\langle L(t)\rangle / k)$. This is given by $\langle L(t)\rangle=$ $\theta / \sum_{r=1}^{\infty} C_{r}(t)$ where

$\sum_{r=1}^{\infty} C_{r}(t)=\frac{\left[1-e^{-t}\right][G(t)-D]}{2-D}+\int_{0}^{t} d u\left[e^{-u}-e^{-k u}\right] G(u)$,

is the average number of islands. For $D=0$ and $D=1$, these results reduce to the standard RSA [17] and ARSA [12] expressions, respectively. Note that, despite the appearance of the factor $1 /(D-2)$ in some of the expressions above, it can be shown, by expanding $F(t)$ in powers of $D-2$, that all the results are well defined and continuous for $D=2$; in particular, $F(t) \rightarrow e^{-t}$ and $G(t) \rightarrow 2$ in the limit $D \rightarrow 2$.

We define the mass-adsorption efficiency, $\epsilon_{m}$, as the ratio of the mass adsorbed to the mass that has collided with the substracte until saturation. We also define the process efficiency, $\epsilon$, as the product of the mass efficiency with the saturation coverage, i.e., $\epsilon=\theta\left(t_{c}\right)^{2} /\left[k \tau_{c}\right]$, which is plotted in Fig. 2.

In the special case of monomer deposition $(k=1)$, we have

$$
\theta(t)=\frac{1-\exp [-(1-D) t]}{1-D}
$$

for $t \leq t_{c}$, an expression first derived in [11]. At the finite time $t_{c}=\ln (1 / D) /(1-D)$ the lattice becomes full $\left(\theta\left(t_{c}\right)=1\right)$. Linear growth $\theta \propto t[11,12]$, typical of some domain-growth models [19], occurs only for $D=1$. When $D<1(>1)$ the sticking probability $d \theta / d t$ decreases (increases) with $\theta$. The average island size for $k=1$ is

$$
\langle L(t)\rangle=\frac{\theta}{1-\theta} \frac{1}{1-e^{-t}} .
$$

This result yields $\langle L\rangle=t /\left[(1-t)\left(1-e^{-t}\right)\right]$ for $D=1$ and $\langle L\rangle=e^{t}$ for $D=0$, and implies that $\langle L\rangle \sim 1 /(1-\theta)$ as $\theta \rightarrow 1$ for all $D$. For dimer deposition $(k=2)$, Eqs.(1-9) simplify, but not sufficiently to allow the coverage to be expressed in a simple closed form for arbitrary $D$.

We now return to discuss the general properties of $k$ mer deposition. For $D>1$, when the growth of islands is preferred to their nucleation, the coverage $\theta$ grows exponentially. In particular, in the scaling regime where $D \rightarrow \infty$ and $t \rightarrow 0$ with $\tau \equiv D t$ fixed, we find that

$$
\theta(t) \approx \frac{1}{D}\left[\frac{2 k-1}{k}\left(e^{k \tau}-1\right)-(k-1) \tau\right]
$$

with $\theta\left(\tau_{c}\right)=1-O(1 / D)$ and $\tau_{c}=(1 / k) \ln [D k /(2 k-$ $1)]+O(1 / D)$. In the limit $D \rightarrow \infty$ there is full coverage because there is no nucleation and, therefore, no gaps smaller than $k$ are created. However, if the initial condition (which needs to contain at least one particle) has a finite fraction of occupied sites, then the latter result may need modification. The divergence of the saturation time, $\tau_{c} \sim \ln (D)$, is a consequence of unsuccessful direct deposition attempts (the rate of success of these events is, after rescaling, $1 / D)$.

In the continuum version of this model, particles of length 1 are deposited on a $1 d$ continuum. This version can be obtained from the lattice model in the same way as the random carparking model is obtained from the lattice RSA model [17], or the continuum ARSA is obtained from its lattice counterpart [12]. This is done by taking $k \rightarrow \infty$ and $t \rightarrow 0$ whilst keeping $\tau \equiv k t$ fixed. Carrying out this procedure gives the following quantities for continuum ARSA with desorption

$$
\begin{aligned}
F(\tau) & =\exp \left[(2-D) \int_{0}^{\tau} d u \frac{1-e^{-u}}{u}\right] \\
G(\tau) & =2 / F(\tau) \\
\theta(\tau) & =\frac{D \tau+2(1-D) \int_{0}^{\tau} d u / F(u)}{2-D}
\end{aligned}
$$

and the saturation time $\tau_{c}$ is the solution of $F\left(\tau_{c}\right)=2 / D$. Note that $\theta$ has the expected forms when $D=0$ and $D=1$.

To summarise, we have derived exact results for $1 d$ deposition with both diffusion and desorption in the prescursor state. Since the random sequential adsorption and accelerated random sequential adsorption are special cases of the current model, previous exact solutions $[12,16,17]$ can be derived from the solution presented here. By changing the desorption rate $D$ we can change between a system in which islands can only nucleate, through a system in which islands can both nucleate and grow, to a system in which islands grow but there is no nucleation. The system saturates for all values of the parameters and the rescaled saturation time $k t_{c}$ exhibits a miniumum at $D=1$ and diverges as $D \rightarrow 0$ and $D \rightarrow \infty$. Conversely, the process efficiency $\epsilon$ has a maximum at $D=1$ and goes to 0 as $D \rightarrow 0$ and $D \rightarrow \infty$. 
In the limit of infinite desorption rate, $D \rightarrow \infty$, the system has an absorbing state $(\theta=0)$, i.e. a state from which the system evolves no further. If the model included desorption from the chemisorbed state, then it would exhibit long-term quasi-equilibrium states and a continuous phase transition between the absorbing state and equilibrium states with $\theta>0$. The limit $D=\infty$ is analogous to an epidemic process in which sites at the edge of a disease cluster are infected at rate proportional to the area of the cluster. In this context, desorption, if present, would correspond to the recovery of infectives [20]; the model would then be similar to the Contact Process [22], but with a rate of infection proportional to area of the neighbouring cluster rather than the number of infected nearest-neighbours.

In practice, two-dimensional models are usually more relevant to physical applications. The $1 d$ system is, nevertheless, useful for obtaining analytic solutions and gaining insight. There are two possible approaches for study- ing this system in $2 d$. One approach is stochastic and spatially-explicit simulation. The other approach is to extend the above equations and to solve them using truncation methods, such as cluster approximations [20?].

A number of further extensions are possible to this model. One of these would be to consider the deposition of mixtures of particles of different sizes, in order to model the situation where the gaseous phase contains a mixture of gases. One could also allow the chemisorbed particles to diffuse in the empty spaces, as was done in [23] where a $1 d$ random sequential adsorption model with diffusing chemisorbed particles was solved exactly. Such extensions will be examined in future work.

JANF was partially supported by the the Scottish Executive Rural Affairs Department (SERAD) while in Edinburgh.
[1] P. J. Flory, J. Am. Chem. Soc. 611518 (1939).

[2] B. Widom, J. Chem. Phys. 443888 (1963); J. Chem. Phys. 554043 (1973).

[3] J. W. Evans, Rev. Mod. Phys. 651281 (1993).

[4] M. A. Morris and M. Bowker and D. A. King, Comprehensive Chemical Kinetics, vol. 19, chapter 1, C. H. Bamford, C. F. H. Tipper and R. G. Compton, eds., Elsevier:Amesterdam (1984).

[5] B. Senger, J.C. Voegel, P. Schaaf, A. Johner, A. Schmitt and J. Talbot, Phys. Rev. A 44, 6926 (1991).

[6] G. Tarjus, P. Schaaf and J. Talbot, J. Chem. Phys. 93 8352 (1990).

[7] R. Jullien and P. Meakin, J. Phys. A 25 L189 (1992); G. Tarjus, P. Viot, H. S. Choi and J. Talbot, Phys. Rev. E 493239 (1994).

[8] P. Kisliuk, J. Phys. C 395 (1957); J. Phys. C 578 (1958).

[9] D. A. King and M. G. Wells, Proc. R. Soc. A 339245 (1974).

[10] D. E. Brown, D. J. Moffat and R. A. Wolkow, Science 279 542 (1998).

[11] J. A. N. Filipe and G. J. Rodgers, Phys. Rev. E 526044 (1995).
[12] G. J. Rodgers and J. A. N. Filipe, J. Phys. A 303449 (1997).

[13] J. A. N. Filipe, G. J. Rodgers and Z. Tavassoli J. Phys. A 317417 (1998).

[14] S. N. Majumdar and D. S. Dean, Phys. Rev. Lett. 89 11571 (2002).

[15] D. E. Knuth, The Art of Computer Programming (Addison-Wesley, Reading, MA, 1998) Vol. 3 2nd ed.

[16] E. R. Cohen and H. Reiss, J. Chem. Phys. 38680 (1963).

[17] J. J. González, P. C. Hemmer and J. S. Høoye, Chem. Phys. 3, 228 (1974)

[18] P. L. Krapivsky, J. Stat. Phys. 69135 (1992).

[19] E. Ben Naim and P. L. Krapivsky, Phys. Rev. E 543562 (1996).

[20] J. A. N. Filipe and G. J. Gibson, Bull. Math. Biol. 63 603 (2001).

[21] R. S. Nord and J. D. Evans, J. Chem. Phys. 82, 2795 (1985).

[22] T. E. Harris, Ann. Prob. 2969 (1974).

[23] S. Kartal and G. J. Rodgers, Phys. Lett. A 225239 (1997). 